\title{
Late-night-dinner is associated with poor glycemic control in people with type 2 diabetes: The KAMOGAWA- DM cohort study
}

\author{
Ryosuke Sakai ${ }^{1)}$, Yoshitaka Hashimoto ${ }^{1)}$, Emi Ushigome ${ }^{1)}$, Akane Miki ${ }^{1)}$, Takuro Okamura' ${ }^{1}$, \\ Masako Matsugasumi ${ }^{1)}$, Takuya Fukuda ${ }^{1)}$, Saori Majima ${ }^{1)}$, Shinobu Matsumoto ${ }^{1)}$, Takafumi Senmaru'), \\ Masahide Hamaguchi ${ }^{2}$, Muhei Tanaka ${ }^{1)}$, Mai Asano' ${ }^{1)}$, Masahiro Yamazaki ${ }^{1)}$, Yohei Oda ${ }^{1)}$ and \\ Michiaki Fukui ${ }^{1)}$ \\ 1) Department of Endocrinology and Metabolism, Kyoto Prefectural University of Medicine, Graduate School of Medical Science, \\ Kyoto, Japan \\ 2) Department of Diabetology, Kameoka Municipal Hospital, Kyoto, Japan
}

\begin{abstract}
Skipping breakfast or irregular breakfast is associated with poor glycemic control. However, a relationship between the timing of dinner and glycemic control in people with type 2 diabetes remains indefinite. Therefore, we investigated the relationship between late-night-dinner and glycemic control in people with type 2 diabetes. We performed questionnaire survey for lifestyle factors in this cross-sectional study. We defined having dinner later than eight pm as late-night-dinner. We examined the differences in clinical and metabolic parameters between those who have late-night-dinner and those who do not have. We also examined the relationship between late-night-dinner and HbAlc, using multiple regression analysis. Ninetyfive people $(23.2 \%)$ had a late-night-dinner, among 409 people with type 2 diabetes. Metabolic parameters (mean (SD) or median (interquartile range)) of people with late-night-dinner were worse than those of without, including body mass index (BMI) (24.4 (4.0) vs. 23.2 (3.4) kg/m², $p=0.006)$, triglycerides (1.5 (1.1-2.1) vs. $1.2(0.8-1.7) \mathrm{mmol} / \mathrm{L}, p<0.001)$, HDLcholesterol (1.4 (0.4) vs. $1.6(0.4) \mathrm{mmol} / \mathrm{L}, p=0.004)$ and hemoglobin Alc (58.1 (13.3) vs. $55.2(10.2) \mathrm{mmol} / \mathrm{mol},(7.5(1.2)$ vs. $7.2(0.9) \%), p=0.023)$ ). Late-night-dinner (standardized regression coefficient $=0.13, p=0.028$ ) was associated with hemoglobin A1c after adjusting for age, BMI, sex, duration of diabetes, smoking, exercise, alcohol, snacking after dinner, nighttime sleep duration, time from dinner to bedtime, skipping breakfast, and medication for diabetes. Late-night-dinner is independently associated with poor glycemic control in people with type 2 diabetes.
\end{abstract}

Key words: Nutrition and diet, Obesity, Lifestyle, Type 2 diabetes, Late-night-dinner

LIFESTYLE CHANGE, which has become diverse and irregular, recently, is the cause of change in food consumption patterns [1,2]. Energy regulation is associated with the circadian clock in molecular level and food intake has important role in metabolic parameters, through circadian clock [3-5]. Irregularity of meal pattern is now reported as a new potential risk factor for

Submitted Sep. 22, 2017; Accepted Dec. 16, 2017 as EJ17-0414 Released online in J-STAGE as advance publication Jan. 27, 2018 Correspondence to: Yoshitaka Hashimoto M.D. Ph.D., Department of Endocrinology and Metabolism, Kyoto Prefectural University of Medicine, Graduate School of Medical Science, 465 Kajii-cho, Kawaramachi-Hirokoji, Kamigyo-ku, Kyoto 602-8566, Japan.

E-mail: y-hashi@koto.kpu-m.ac.jp metabolic syndrome, diabetes and cardiovascular disease $[6,7]$. In fact, irregular and skipping breakfast increase the risk of obesity, metabolic syndrome and poor glycemic control [8].

Meanwhile, in Japan, dinner is the main meal of the day and the greatest energy is intake by dinner [9]. About two-fifths Japanese eat dinner from 2000 to 2400 $\mathrm{h}$ [10]. It has been reported that late-night-dinner has associated with obesity, metabolic syndrome and hyperglycemia in general population [11-14]. Besides, it is reported that the risks of metabolic syndrome, diabetes and insulin resistance in the people with having high calorie at dinner were higher than those in the people with having high calorie at breakfast [15]. Another study 
showed that the evening chronotype was risk of diabetes and metabolic syndromes [16]. In fact, it is well known that night shift work is a risk of incident diabetes [17, 18]. Thus, dinner timing is an important issue in the clinical setting. However, little is known about the relationship between late-night-dinner and glycemic control in people with type 2 diabetes. Therefore, in this crosssectional study, we examined the relationship between late-night-dinner and glycemic control of people with type 2 diabetes.

\section{Materials and Methods}

\section{Patients and study design}

The KAMOGAWA-DM cohort study is an ongoing cohort study of people with diabetes. The purpose of this cohort study, which was started from 2014, is to clarify the natural history of people with diabetes. We served the results of the medical data into a database after giving informed consent and keeping personal identifiable information secret. The inclusion criteria of this crosssectional study was the outpatient with type 2 diabetes, diagnosed by a physician, at the Kyoto Prefectural University of Medicine, from January 2014 to January 2016. The people under steroid treatment, after a renal transplantation, after a liver transplantation, with severe renal dysfunction (estimated Glomerular filtration rate (eGFR) $<30 \mathrm{~mL} / \mathrm{min} / 1.73 \mathrm{~m}^{2}$ ) [19], shift workers who work at night and the people who did not have dinner were excluded. In addition, the people, whose data (blood biochemical examinations or questionnaires) were incomplete, were also excluded. Approval for this study was obtained from the Ethics Committee of Kyoto Prefectural University of Medicine (No. RBMR-E-466-1).

\section{Questionnaire and measurements}

We evaluated lifestyle factors by a questionnaire survey. As for exercise status, the people who performed any kind of sports at least once a week regularly on the questionnaire were defined as regular exercisers [20]. As for alcohol, the people who drank alcohol daily were defined as alcohol drinker. As for smoking, smoking status was defined by current use of cigarette [21, 22]. Skipping breakfast was determined based on a negative answer to the question: "Do you have breakfast regularly?" In addition, we asked whether having daily dinner or not and the time of dinner. When the answer was having daily dinner and the dinner time was later than eight pm [23], we defined it as late-night-dinner. About status of snacking after dinner, we defined it as having daily snacking after dinner by the answer of the regular snacking time and dinner time. Nighttime sleep duration was evaluated by the answer of the regular bedtime and the regular get up time, and likewise the time from dinner to bedtime was evaluated by the answer of the regular bedtime and the dinner time.

Body mass index (BMI) was defined as weight in kilograms divided by height in meters squared. Overweight and obesity status were defined as BMI over $23 \mathrm{~kg} / \mathrm{m}^{2}$ and over $25 \mathrm{~kg} / \mathrm{m}^{2}$ [24-26]. Venous blood after an overnight fast were collected and then we measured the levels of various factors, including fasting plasma glucose, aspartate aminotransferase, alanine aminotransferase, gamma-glutamyltransferase, total cholesterol, triglycerides, high-density lipoprotein (HDL) cholesterol, uric acid and creatinine. Hemoglobin Alc was assayed using high-performance liquid chromatography and was expressed as a National Glycohemoglobin Standardization Program unit. Glomerular filtration rate (GFR) was estimated using the Japanese Society of Nephrology equation: estimated GFR (eGFR) $\left(\mathrm{mL} / \mathrm{min} / 1.73 \mathrm{~m}^{2}\right)=$ $194 \times$ serum creatinine $\mathrm{e}^{-1.094} \times$ age $^{-0.287}(\times 0.739$ for women $)$ [27].

We also investigated the diabetic medications and categorized as follows: insulin, insulin secretagogues, insulin sensitizers and nutrient load reducers [28]. Insulin secretagogues included sulfonylureas, glinides, dipeptidyl peptidase-4 inhibitors and glucagon-like peptide-1 receptor agonist; insulin sensitizers included pioglitazone and metformin; and nutrient load reducers included $\alpha$ glucosidase inhibitors and sodium-glucose cotransporter inhibitors [28]. Moreover, we also investigated the medications affecting to lipids and BMI, including statins, fibrates, ezetimibe and eicosapentaenoic acid for lipids and diuretics for BMI.

\section{Statistical analysis}

We used JMP version 12.0 software (SAS Institute, Cary, NC, USA) for statistical analyses and we considered $p<0.05$ as statistically significant. We calculated mean, median and frequencies of potential confounding variables. The people were divided into two groups according to late-night-dinner or not. Continuous variables were presented as the mean (standard deviation (SD)) or median (interquartile range), and Student's $t$ test or Mann-Whitney U test was used for evaluating statistical significance of differences. Categorical variables were presented as percent (number) and Chi-square 


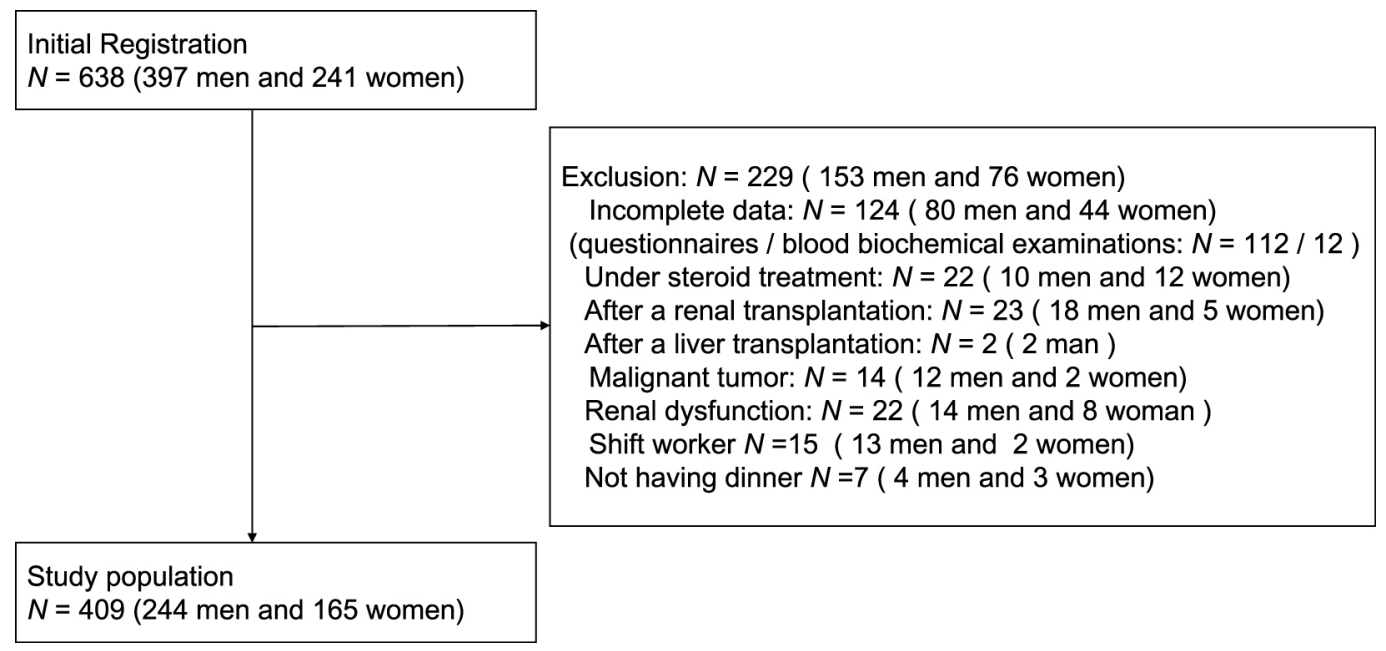

Fig. 1 Inclusion and exclusion of the study participants

test was used for evaluating statistical significance of differences between groups.

To investigate the relationship between various factors and hemoglobin A1c, we performed the multiple regression analysis and the following factors, which are including the well-known variates to affect HbA1c [29-31], were considered simultaneously as independent variables for multiple regression analysis: sex, BMI, age, duration of diabetes, nighttime sleep duration, time from dinner to bedtime, exercise, smoking, snacking after dinner, alcohol drinking, skipping breakfast, and usage of insulin treatment, insulin secretagogues, insulin sensitizers and nutrient load reducers. We also performed the multiple regression analysis to investigate the relationship between late-night-dinner and Log TG, HDL cholesterol or BMI.

\section{Results}

In this study, 638 people (397 men and 241 women) with type 2 diabetes were included. Among them, 229 people (153 men and 76 women) were excluded; 112 people who were incomplete with questionnaires, and 12 people who were with blood biochemical examinations were included. Thus, the study population was 409 people (244 men and 165 women) (Fig. 1).

Clinical characteristics of study participants are shown in Table 1. The average (SD) of age and duration of diabetes were 68.3 (10.9) years and $13.6(10.1)$ years. In this study population, 95 people $(23.2 \%)$ had late-nightdinner, 17 people (4.2\%) skipped breakfast, and 22 peo- ple (5.3\%) had regularly snacking after dinner.

Metabolic parameters, including BMI, blood pressure, $\mathrm{HbAlc}$, total cholesterol, triglycerides, alanine aminotransferase and gamma-glutamyltransferase, of people with late-night-dinner were higher than those of people without and HDL cholesterol of people with late-nightdinner was lower than that of people without (Table 1).

The ratio of exerciser in people with late-night-dinner was lower than that in people without $(p=0.015)$ and the ratio of overweight, obesity and skipping breakfast in people with late-night-dinner was higher than that in people without ( $p=0.003$ ) (Table 1). In addition, we also investigated the dose and frequency of drug including glimepiride $(n=130)$, metformin $(n=139)$, sitagliptin $(n$ $=128)$ and voglibose $(n=24)$, which were mainly prescribed in each class, and insulin $(n=87)$, and we compered them between people with and without latenight-dinner. Among them, average dose of insulin was solely different between people with and without latenight-dinner (25.9 (12.8) vs. 18.9 (11.9) units, $p=0.027$ ).

Multiple regression analysis showed that late-nightdinner (standardized regression coefficient $=0.131, p=$ 0.026) was associated with $\mathrm{HbA} 1 \mathrm{c}$ after adjusting covariates (Table 2). In addition to that, dinner time (as a continuous variable) was associated with $\mathrm{HbAlc}$ (standardized regression coefficient $=0.141, p=0.029$ ) after adjusting covariates. Furthermore, late-night-dinner was associated with Log TG (standardized regression coefficient $=0.116, p=0.039$ ) after adjusting for sex, BMI, age, duration of diabetes, nighttime sleep duration, time from dinner to bedtime, exercise, smoking, snacking 
Table 1 Clinical characteristics of study patients

\begin{tabular}{|c|c|c|c|c|}
\hline & Total & Late-night-dinner (-) & Late-night-dinner $(+)$ & $p$ \\
\hline$N$ & 409 & 314 & 95 & - \\
\hline Men/Women $(n)$ & $244 / 165$ & $176 / 138$ & $68 / 27$ & 0.007 \\
\hline Age (year) & $68.3(10.9)$ & $70.6(9.3)$ & $60.7(12.3)$ & $<0.001$ \\
\hline Duration of diabetes (year) & $13.6(10.1)$ & $13.9(10.1)$ & $12.5(10.2)$ & 0.156 \\
\hline Body mass index $\left(\mathrm{kg} / \mathrm{m}^{2}\right)$ & $23.5(3.6)$ & $23.2(3.4)$ & $24.4(4.0)$ & 0.006 \\
\hline Non-overweight/Overweight/Obesity $(n)$ & $180 / 117 / 112$ & $151 / 86 / 77$ & $29 / 31 / 35$ & 0.007 \\
\hline Systolic blood pressure (mmHg) & $129.7(15.0)$ & $129.5(15.3)$ & $130.6(14.1)$ & 0.497 \\
\hline Diastolic blood pressure $(\mathrm{mmHg})$ & $71.8(11.5)$ & $70.5(10.9)$ & $76.1(12.2)$ & $<0.001$ \\
\hline Hemoglobin A1c (mmol/mol) & $55.9(11.0)$ & $55.2(10.2)$ & $58.1(13.3)$ & 0.023 \\
\hline Hemoglobin A1c (\%) & $7.3(1.0)$ & $7.2(0.9)$ & $7.5(1.2)$ & 0.023 \\
\hline Fasting plasma glucose $(\mathrm{mmol} / \mathrm{L})$ & $8.1(2.5)$ & $8.1(2.4)$ & $8.3(2.7)$ & 0.560 \\
\hline Total cholesterol $(\mathrm{mmol} / \mathrm{L})$ & $4.8(0.8)$ & $4.7(0.8)$ & $5.0(1.0)$ & 0.011 \\
\hline Triglycerides (mmol/L) & $1.2(0.9-1.7)$ & $1.2(0.8-1.7)$ & $1.5(1.1-2.1)$ & $<0.001$ \\
\hline HDL cholesterol (mmol/L) & $1.5(0.4)$ & $1.6(0.4)$ & $1.4(0.4)$ & 0.004 \\
\hline Aspartate aminotransferase (IU/L) & $25.6(13.5)$ & $25.1(11.8)$ & $27.0(17.9)$ & 0.851 \\
\hline Alanine aminotransferase (IU/L) & $24.4(18.7)$ & $22.7(15.9)$ & $29.7(25.2)$ & 0.002 \\
\hline gamma-glutamyltransferase (IU/L) & $25(18-41)$ & $24(17-37)$ & $30(21-52)$ & 0.001 \\
\hline Creatinine $(\mu \mathrm{mol} / \mathrm{L})$ & $70.6(21.7)$ & $70.4(22.1)$ & $71.3(20.2)$ & 0.731 \\
\hline $\operatorname{eGFR}\left(\mathrm{mL} / \mathrm{min} / 1.73 \mathrm{~m}^{2}\right)$ & $71.7(19.1)$ & $70.5(18.7)$ & $75.9(19.9)$ & 0.022 \\
\hline Uric acid $(\mu \mathrm{mol} / \mathrm{L})$ & $313(76)$ & $311(77)$ & $319(76)$ & 0.427 \\
\hline Nighttime sleep duration (hour) & $7.3(1.4)$ & $7.5(1.4)$ & $6.8(1.3)$ & $<0.001$ \\
\hline Time from dinner to bedtime (hour) & $4.3(1.3)$ & $4.5(1.2)$ & $3.5(1.2)$ & $<0.001$ \\
\hline Exercise $(-/+)$ & $188 / 221$ & $134 / 180$ & $54 / 41$ & 0.015 \\
\hline Smoking $(-/+)$ & $357 / 52$ & $278 / 36$ & $79 / 16$ & 0.168 \\
\hline Alcohol drinking $(-/+)$ & $323 / 86$ & $248 / 66$ & $75 / 20$ & 0.994 \\
\hline Snacking after dinner $(-/+)$ & $387 / 22$ & $297 / 17$ & $90 / 5$ & 0.955 \\
\hline Skipping breakfast $(-/+)$ & $392 / 17$ & $306 / 8$ & $86 / 9$ & 0.003 \\
\hline Late-night-dinner $(-/+)$ & $314 / 95$ & - & - & - \\
\hline Insulin treatment $(-/+)$ & $317 / 92$ & $241 / 73$ & $76 / 19$ & 0.506 \\
\hline Insulin dose (units/ day) & $20.4(12.4)$ & $18.9(11.9)$ & $25.9(12.8)$ & 0.027 \\
\hline Diuretics $(-/+)$ & $345 / 64$ & $261 / 53$ & $84 / 11$ & 0.213 \\
\hline Statins $(-/+)$ & $219 / 190$ & $166 / 148$ & $53 / 42$ & 0.617 \\
\hline Fibrates $(-/+)$ & $385 / 24$ & $300 / 14$ & $85 / 10$ & 0.028 \\
\hline Ezetimibe $(-/+)$ & $390 / 19$ & $297 / 17$ & $93 / 2$ & 0.179 \\
\hline Eicosapentaenoic acid $(-/+)$ & $398 / 11$ & $308 / 6$ & $90 / 5$ & 0.077 \\
\hline Insulin secretagogues $(-/+)$ & $100 / 309$ & $79 / 235$ & $21 / 74$ & 0.544 \\
\hline Sulfonylureas & $235 / 174$ & $177 / 137$ & $58 / 37$ & 0.419 \\
\hline Glinides & $376 / 33$ & $287 / 27$ & $89 / 6$ & 0.474 \\
\hline Dipeptidyl peptidase- 4 inhibitors & $172 / 237$ & $132 / 182$ & $40 / 55$ & 0.991 \\
\hline Glucagon-like peptide-1 receptor agonist & $387 / 22$ & $302 / 12$ & $85 / 10$ & 0.011 \\
\hline Insulin sensitizers $(-/+)$ & $249 / 160$ & $198 / 116$ & $51 / 44$ & 0.101 \\
\hline Pioglitazone & $378 / 31$ & $287 / 27$ & $91 / 4$ & 0.157 \\
\hline Metformin & $266 / 143$ & $214 / 100$ & $52 / 43$ & 0.016 \\
\hline Nutrient load reducers $(-/+)$ & $335 / 74$ & $255 / 59$ & $80 / 15$ & 0.506 \\
\hline$\alpha$-glucosidase inhibitors & $357 / 52$ & $274 / 40$ & $83 / 12$ & 0.978 \\
\hline Sodium-glucose cotransporter inhibitors & $385 / 24$ & $294 / 20$ & $91 / 4$ & 0.433 \\
\hline
\end{tabular}

Data are expressed as mean (SD), median (interquartile range) or number. HDL, High-density lipoprotein; eGFR, estimated glomerular filtration rate. The difference between groups was analyzed by Student's $t$-test, Mann-Whitney U test or Chi-square test. 
Table 2 Multiple regression analysis on Hemoglobin A1c (mmol/ mol)

\begin{tabular}{|c|c|c|}
\hline & $\begin{array}{c}\text { standardized } \\
\text { regression coefficient }\end{array}$ & $p$ \\
\hline Sex & -0.046 & 0.365 \\
\hline Age (year) & 0.038 & 0.506 \\
\hline Duration of diabetes (year) & 0.165 & 0.002 \\
\hline Body mass index $\left(\mathrm{kg} / \mathrm{m}^{2}\right)$ & 0.185 & 0.000 \\
\hline Exercise & -0.029 & 0.549 \\
\hline Smoking & 0.034 & 0.478 \\
\hline Alcohol drinking & -0.026 & 0.605 \\
\hline Skipping breakfast & 0.032 & 0.516 \\
\hline Snacking after dinner & -0.035 & 0.464 \\
\hline Insulin treatment & 0.130 & 0.012 \\
\hline Insulin secretagogues & 0.103 & 0.035 \\
\hline Insulin sensitizers & 0.041 & 0.403 \\
\hline Nutrient load reducers & 0.076 & 0.119 \\
\hline $\begin{array}{l}\text { Time from dinner to bedtime, } \\
\text { hour }\end{array}$ & 0.092 & 0.131 \\
\hline Nighttime sleep duration, hour & -0.047 & 0.413 \\
\hline Late-night-dinner & 0.129 & 0.028 \\
\hline
\end{tabular}

Sex was defined as women $(=0)$ or men $(=1)$; exercise status was defined as non-regular exerciser $(=0)$ or regular exerciser $(=1)$; smoking status was defined as non-smoker $(=0)$ or smoker $(=1)$; alcohol drinking was defined as non-regular alcohol consumer $(=0)$ or regular alcohol consumer $(=1)$; skipping breakfast was defined as non-skipper $(=0)$ or skipper $(=1)$; snacking after dinner was defined as not having daily snacking after dinner $(=0)$ or having $(=1)$; insulin treatment, insulin secretagogues, insulin sensitizers and nutrient load reducers were defined as without $(=0)$ or with $(=1)$. Late-night-dinner was determined by the dinner time. We defined the late-night-dinner which is having dinner later than 20:00 as without $(=0)$ or with $(=1)$.

after dinner, alcohol drinking, skipping breakfast, and usage of statins, fibrates, ezetimibe and eicosapentaenoic acid for lipids, whereas, late-night-dinner was not associated with HDL cholesterol (standardized regression coefficient $=-0.056, p=0.312$ ). Moreover, late-nightdinner was not associated with BMI (standardized regression coefficient $=0.080, p=0.155$ ) after adjusting for sex, age, duration of diabetes, nighttime sleep duration, time from dinner to bedtime, exercise, smoking, snacking after dinner, alcohol drinking, skipping breakfast and diuretics affecting to BMI.

\section{Discussion}

We demonstrated that late-night-dinner was independently associated with poor glycemic control in people with type 2 diabetes. In addition, we also demonstrated that the metabolic parameters, especially TG, of people with late-night-dinner were worse than those of people without. It has been reported that late-night-dinner is associated with poor cardiometabolic profiles in general population [12, 32]. However, the relationship between late-night-dinner and glycemic control in people with type 2 diabetes is not reported. This is the first study to clarify the relationship between late-night-dinner and glycemic control in people with type 2 diabetes.

One of the possible explanations why late-night-dinner is associated with poor glycemic control is that latenight-dinner prolongs the postprandial glucose spike for a long time [33]. In fact, during the night, decreasing of glucose tolerance and insulin sensitivity are shown [34]. Another possible explanations is the effect of circadian rhythms on glucose metabolism. It is well known that many physiologically and nutritionally processes have the circadian rhythms in human body [35]. Nutrition factors, such as food intake, appetite and digestion, have an effect on circadian patterns [36]. Food intake modulates the circadian rhythms, especially in liver and intestine $[37,38]$ and misalignment of circadian rhythm leads to insulin resistance and diabetes [39, 40]. In fact, skipping breakfast, one of the irregular eating, is associated with metabolic syndrome, diabetes and cardiovascular disease $[6,7]$. Moreover, late-night-dinner might be concerned with poor glycemic control via reduced diet-induced thermogenesis (DIT). Because, DIT is about $10 \%$ of total energy expenditure, and it is the greatest in the morning and declines toward late at night [41]. Taking together, late-night-dinner is associated with poor glycemic control.

We also demonstrated that duration of diabetes, insulin treatment and usage of insulin secretagogues were also associated with poor glycemic control in this study. It has been reported that usage of insulin or number of antidiabetic medications was associated with poor glycemic control $[29,30]$. In fact, usage of insulin secretagogues was correlated with number of antidiabetic medications ( $p<0.001$ by Student's $t$-test) in this study. Medication is the important treatment for type 2 diabetes, but diet and exercise therapies are the fundamental treatments for patients with type 2 diabetes [42]. Therefore, we often fail to adjust $\mathrm{HbAlc}$ by modification of medication, including due to poor adherence of medication [43], 
although the details of adherence of medication in people with diabetes is not clarified.

In this study, we demonstrated that late-night-dinner was a risk of poor glycemic control. Thus, to improve glycemic control, having dinner before eight $\mathrm{pm}$ is needed. However, it is difficult for all people to have dinner before eight $\mathrm{pm}$. A recent study demonstrated that dividing consumption of late-night-dinner can suppress postprandial hyperglycemia [44].

This study has some limitations. First, we evaluated dinner time and lifestyle factors using self-reports. There is a potential bias, because there is a possibility that people may report generally desirable answers which are considered to be healthy. In addition, the validity of a questionnaire for late-night-dinner is not enough. However, many studies have defined nighttime dinners using a questionnaire including answers to dinner time $[12,45$, 46]. Likewise, we assessed late-night-dinner as the major observation by the time obtained from questionnaire. Second, we did not have the detail data of the meal. It is reported that the late-night-dinner is less satiating and can become the cause of overeating [47]. Therefore, the overeating as a result of late-night-dinner might have affected the poor glycemic control. However, even with the same meal contents, consuming in late-night-dinner lead to postprandial hyperglycemia [44]. Furthermore, it is reported that there is a significant correlation between skipping breakfast and late-night-dinner [48]. So, skipping breakfast might affect the result on this study. In fact, the proportion of skipping breakfast in people with late-night-dinner was higher than that in people without $(p=0.003)$ in this study. However, when we used a skipping breakfast as a covariate in this study, the effect of late-night-dinner was independently associated with worse $\mathrm{HbAlc}$ from skipping breakfast. In addition, the glycemic control of people with late-night-dinner were worse than that of people without $(7.43 \%$ vs. $7.20 \%, p=$ 0.047 by Chi-square test), even when we excluded people who regularly skipped breakfast. Thus, late-nightdinner itself is associated with the poor glycemic control. Third, medications for diabetes might affect the results of this study, although we handled the medications for diabetes as covariates to correct the effect of medications in this study. Lastly, in this study, people are Japanese only. Therefore, the generalizability of our study to nonJapanese populations is uncertain.

In conclusion, this cross-sectional study demonstrates, for the first time, late-night-dinner is associated with poor glycemic control in people with type 2 diabetes.
Thus, we should focus more on the timing of dinner for management of people with type 2 diabetes.

\section{Acknowledgements}

All the authors contributed to this manuscript. RS designed the study, researched data and wrote manuscript. YH originated and designed the study, researched data and reviewed the manuscript. EU originated the study, researched data and contributed to discussion. AM, TO, MM, TF, SaM, ShM, and TS researched data and contributed to discussion. MH, MT, MA, and MY originated the study and contributed to discussion. MF researched data and reviewed and edited the manuscript. $\mathrm{YH}$ is the guarantor of this work and, as such, had full access to all the data in the study and takes responsibility for the integrity of the data and the accuracy of the data analysis.

\section{Funding Sources}

None.

\section{Disclosure}

Honoraria; Masahiro Yamazaki from AstraZeneca plc. Research funding; Mai Asano from Ono Pharmaceutical Co, LTD. Fees for promotional materials; Michiaki Fukui; AstraZeneca plc., Astellas Pharma Inc., Nippon Boehringer Ingelheim Co., Ltd., Daiichi Sankyo Co., Ltd., Eli Lilly Japan K.K., Kyowa Hakko Kirin Company Ltd., Kissei Pharmaceutical Co., Ltd., MSD K.K., Mitsubishi Tanabe Pharma Corporation, Novo Nordisk Pharma Ltd., Sanwa Kagaku Kenkyusho Co., Ltd., Sanofi K.K., Ono Pharmaceutical Co., Ltd., and Takeda Pharmaceutical Co., Ltd. The sponsors were not involved in the study design; in the collection, analysis, interpretation of data; in the writing of this manuscript; or in the decision to submit the article for publication. The authors, their immediate families, and any research foundations with which they are affiliated have not received any financial payments or other benefits from any commercial entity related to the subject of this article. The authors declare that although they are affiliated with a department that is supported financially by pharmaceutical company, the authors received no current funding for this study and this does not alter their adherence to all the journal policies on sharing data and materials. The other authors have nothing to disclose. 


\section{References}

1. Samuelson G (2000) Dietary habits and nutritional status in adolescents over Europe. An overview of current studies in the Nordic countries. Eur J Clin Nutr 54: S21-S28.

2. Bertéus Forslund H, Torgerson JS, Sjöström L, Lindroos AK (2005) Snacking frequency in relation to energy intake and food choices in obese men and women compared to a reference population. Int J Obes (Lond) 29: 711-719.

3. Arble DM, Bass J, Laposky AD, Vitaterna MH, Turek FW (2009) Circadian timing of food intake contributes to weight gain. Obesity (Silver Spring) 17: 2100-2102.

4. Turek FW, Joshu C, Kohsaka A, Lin E, Ivanova G, et al. (2005) Obesity and metabolic syndrome in circadian clock mutant mice. Science 308: 1043-1045.

5. Kohsaka A, Laposky AD, Ramsey KM, Estrada C, Joshu C, et al. (2007) High-fat diet disrupts behavioral and molecular circadian rhythms in mice. Cell Metab 6: 414421.

6. Garaulet M, Madrid JA (2010) Chronobiological aspects of nutrition, metabolic syndrome and obesity. Adv Drug Deliv Rev 62: 967-978.

7. Tahara Y, Shibata S (2013) Chronobiology and nutrition. Neuroscience 253: 78-88.

8. Jakubowicz D, Wainstein J, Ahren B, Landau Z, BarDayan Y, et al. (2015) Fasting until noon triggers increased postprandial hyperglycemia and impaired insulin response after lunch and dinner in individuals with type 2 diabetes: a randomized clinical trial. Diabetes Care 38: $1820-1826$.

9. Takahashi T, Tomizawa M, Ito K, Morino M, Uenishi K, et al. (2008) The distribution of energy intake in a day for married male workers living in metropolitan areas. Journal of Japanese Society of Nutrition and Food Science 61: 273-283 (In Japanese).

10. NHK Broadcasting Culture Research Institute (2015) Japanese Time Use: The NHK National Time Use Survey. https://www.nhk.or.jp/bunken/research/yoron/pdf/ 20160217_1.pdf, Accessed 6 Jannuary 2017 (In Japanese).

11. Wang JB, Patterson RE, Ang A, Emond JA, Shetty N, et al. (2014) Timing of energy intake during the day is associated with the risk of obesity in adults. J Hum Nutr Diet 27: 255-262.

12. Baron KG, Reid KJ, Horn LV, Zee PC (2013) Contribution of evening macronutrient intake to total caloric intake and body mass index. Appetite 60: 246-251.

13. Nakajima K, Suwa K (2015) Association of hyperglycemia in a general Japanese population with late-nightdinner eating alone, but not breakfast skipping alone. $J$ Diabetes Metab Disord 14: 16.

14. Kajiyama S, Imai S, Hashimoto Y, Yamane C, Miyawaki
T, et al. (2017) Divided consumption of late-night-dinner improves glucose excursions in young healthy women: a randomized cross-over clinical trial. Diabetes Res Clin Pract in press. doi: 10.1016/j.diabres.2017.11.033

15. Jakubowicz D, Barnea M, Wainstein J, Froy O (2013) High Caloric intake at breakfast vs. dinner differentially influences weight loss of overweight and obese women. Obesity (Silver Spring) 21: 2504-2512.

16. Yu JH, Yun C-H, Ahn JH, Suh S, Cho HJ, et al. (2015) Evening chronotype is associated with metabolic disorders and body composition in middle-aged adults. J Clin Endocrinol Metab 100: 1494-1502.

17. Hansen AB, Stayner L, Hansen J, Andersen ZJ (2016) Night shift work and incidence of diabetes in the Danish nurse cohort. Occup Environ Med 73: 262-268.

18. Morris CJ, Purvis TE, Mistretta J, Scheer FAJL (2016) Effects of the internal circadian system and circadian misalignment on glucose tolerance in chronic shift workers. $J$ Clin Endocrinol Metab 101: 1066-1074.

19. Webster AC, Nagler EV, Morton RL, Masson P (2017) Chronic kidney disease. Lancet 389: 1238-1252.

20. Hashimoto Y, Hamaguchi M, Kojima T, Ohshima Y, Ohbora A, et al. (2015) Modest alcohol consumption reduces the incidence of fatty liver in men: a populationbased large-scale cohort study. J Gastroenterol Hepatol 30: 546-552.

21. Hashimoto $Y$, Tanaka M, Kimura T, Kitagawa N, Hamaguchi M, et al. (2015) Hemoglobin concentration and incident metabolic syndrome: a population-based large-scale cohort study. Endocrine 50: 390-396.

22. Hashimoto $Y$, Tanaka M, Okada H, Senmaru T, Hamaguchi M, et al. (2015) Metabolically healthy obesity and risk of incident CKD. Clin J Am Soc Nephrol 10: 578-583.

23. Sandhu SK, Tang TS (2017) When's dinner? Does timing of dinner affect the cardiometabolic risk profiles of SouthAsian Canadians at risk of diabetes? Diabet Med 34: 539542.

24. Hashimoto Y, Hamaguchi M, Kojima T, Fukuda T, Ohbora A, et al. (2017) Nonoverweight nonalcoholic fatty liver disease and incident cardiovascular disease: a post hoc analysis of a cohort study. Medicine (Baltimore) 96: e6712.

25. Hashimoto Y, Hamaguchi M, Fukuda T, Ohbora A, Kojima T, et al. (2017) Fatty liver as a risk factor for progression from metabolically healthy to metabolically abnormal in non-overweight individuals. Endocrine 57: 89-97.

26. Hashimoto Y, Hamaguchi M, Fukuda T, Obora A, Kojima $\mathrm{T}$, et al. (2017) Weight gain since age of 20 as risk of 
metabolic syndrome even in non-overweight individuals. Endocrine 58: 253-261.

27. Matsuo S, Imai E, Horio M, Yasuda Y, Tomita K, et al. (2009) Revised equations for estimated GFR from serum creatinine in Japan. Am J Kidney Dis 53: 982-992.

28. Kalra S (2016) Classification of non-insulin glucose lowering drugs. J Pak Med Assoc 66: 1497-1498.

29. Badedi M, Solan Y, Darraj H, Sabai A, Mahfouz M, et al. (2016) Factors associated with long-term control of type 2 diabetes mellitus. J Diabetes Res 2016: 2109542.

30. Cramer JA, Pugh MJ (2005) The influence of insulin use on glycemic control: How well do adults follow prescriptions for insulin? Diabetes Care 28: 78-83.

31. Hashimoto Y, Tanaka M, Yamazaki M, Nakano K, Ushigome E, et al. (2016) Caffeine intake enhances the benefits of sodium glucose transporter 2 inhibitor. Diabetes Metab Res Rev 32: 694-699.

32. Lesser IA, Gasevic D, Lear SA (2014) The association between acculturation and dietary patterns of South Asian immigrants. PLoS One 9: e88495.

33. Sato M, Nakamura K, Ogata H, Miyashita A, Nagasaka S, et al. (2011) Acute effect of late evening meal on diurnal variation of blood glucose and energy metabolism. Obes Res Clin Pract 5: e169-e266.

34. Van Cauter E, Désir D, Decoster C, Féry F, Balasse EO (1989) Nocturnal decrease in glucose tolerance during constant glucose infusion. J Clin Endocrinol Metab 69: 604-611.

35. Johnston JD (2014) Physiological links between circadian rhythms, metabolism and nutrition. Exp Physiol 99: 11331137.

36. Waterhouse J, Minors D, Atkinson G, Benton D (1997) Chronobiology and meal times: internal and external factors. Br J Nutr 77 Suppl 1: S29-S38.

37. Hara R, Wan K, Wakamatsu H, Aida R, Moriya $\mathrm{T}$, et al. (2001) Restricted feeding entrains liver clock without participation of the suprachiasmatic nucleus. Genes Cells 6: 269-278.

38. Froy O, Chapnik N, Miskin R (2005) Mouse intestinal cryptdins exhibit circadian oscillation. FASEB J 19: 19201922.

39. Morris CJ, Yang JN, Scheer FA (2012) The impact of the circadian timing system on cardiovascular and metabolic function. Prog Brain Res 199: 337-358.

40. Marcheva B, Ramsey KM, Buhr ED, Kobayashi Y, Su H, et al. (2010) Disruption of the clock components CLOCK and BMAL1 leads to hypoinsulinaemia and diabetes. Nature 466: 627-631.

41. Morris CJ, Garcia JI, Myers S, Yang JN, Trienekens N, et al. (2015) The human circadian system has a dominating role in causing the morning/evening difference in dietinduced thermogenesis. Obesity (Silver Spring) 23: 20532058.

42. Hamdy O, Goodyear LJ, Horton ES (2001) Diet and exercise in type 2 diabetes mellitus. Endocrinol Metab Clin North Am 30: 883-907.

43. Brown MT, Bussell JK (2011) Medication adherence: WHO cares? Mayo Clin Proc 86: 304-314.

44. Imai S, Kajiyama S, Hashimoto Y, Yamane C, Miyawaki $\mathrm{T}$, et al. (2017) Divided consumption of late-night-dinner improves glycemic excursions in patients with type 2 diabetes: a randomized cross-over clinical trial. Diabetes Res Clin Pract 129: 206-212.

45. Ozawa K, Suzuki A, Takaizumi K, Iwabe M, Matsuki H, et al. (2016) Late night meals and obesity: a systematic review using English-language literature. JJHEP 24: 205216 (In Japanese)

46. Baron KG, Reid KJ, Kern AS, Zee PC (2011) Role of sleep timing in caloric intake and BMI. Obesity (Silver Spring) 19: 1374-1381.

47. de Castro JM (2004) The time of day of food intake influences overall intake in humans. J Nutr 134: 104-111.

48. Kutsuma A, Nakajima K, Suwa K (2014) Potential association between breakfast skipping and concomitant late-night-dinner eating with metabolic syndrome and proteinuria in the Japanese population. Scientifica (Cairo) 2014: 253581. 